\title{
Cost-effectiveness of a Nurse-led ORIF Ankle Care Programme
}

\author{
Maria Cristina Martin \\ University of Strathclyde \\ Computer and Information Sciences \\ cristina.martin@strath.ac.uk
}

\author{
Kerem Akartunalı \\ University of Strathclyde \\ Department of Management Science \\ kerem.akartunali@strath.ac.uk
}

\author{
Gillian Anderson \\ University of Strathclyde \\ Department of Management Science \\ gillian.anderson.101@strath.ac.uk
}

\begin{abstract}
In response to current outdated models of outpatient fracture care, a nurse-led ankle care protocol was implemented by Glasgow Royal Infirmary's (GRI) fracture clinic. Its aim was to standardise postsurgery care for Open Reduction Internal Fixation (ORIF) ankle fractures, while maintaining patient reported outcomes. The demand for evaluation across healthcare in the UK is exponentially increasing and although the protocol has been widely accepted throughout the clinic, no evidence existed to confirm its cost-effectiveness. This study fills that gap in knowledge through a thorough cost-evaluation using Discrete Event Simulation (DES), a widely recognised and powerful modelling tool within healthcare evaluation. It was found that the difference between the total number of appointments attended patients between the two groups was not significant $(p>0.05)$. However, results of the cost-modelling clearly show that a $28.12 \%$ saving can be achieved when comparing total staffing costs and X-ray costs between the two groups.
\end{abstract}

Economic evaluation. Nurse-led clinics. Open Reduction Internal Fixation of the ankle.

\section{INTRODUCTION}

Changes in the training that medical staff receive and the structure of the workforce at orthopaedic fracture clinics have been observed along with a lack of response to these changes in the clinical guidelines set by the British Medical Association [1]. Hence many of the current models for out-patient fracture care are outdated. In response, some of the processes in the out-patient care of minor orthopaedic trauma have been investigated. Glasgow's Royal Infirmary in Scotland has been focused on improving their management, progressively introducing changes leading up to their official service redesign launched in 2010 [1]. Naturally, a demand for evaluation stems from these changes.

Evaluation in healthcare has a crucial role in its continuous improvement. It forms part of a learning curve that enables healthcare professionals to improve existing programs as well developing new ones. Furthermore, healthcare decision-makers are increasingly pushed towards continuous quality improvement. Hoffmann, et al. [2] point out that the inclusion of findings of healthcare evaluations such as cost-effectiveness findings in commissioned reviews by national regulatory bodies such as the NICE (National Institute for Clinical Excellence) acknowledges the importance of such evaluations at the national policy level.
Since the 2009 spending review, the National Health Service has been under tremendous pressure to achieve savings of $£ 15$ to $£ 20$ billion [3]. Hence careful consideration of the effectiveness of healthcare programs is essential, particularly with an economic perspective.

\section{BACKGROUND}

Open Reduction Internal Fixation (ORIF) of the ankle is a common surgical procedure to fix a severe ankle fracture by repositioning of the bones with steel screws or plates. Traditional out-patient care for this type of procedure follows general guidelines and is primarily consultant-led, however, there is a lack of standardisation. In 2005, Glasgow Royal Infirmary's (GRI) fracture clinic implemented a nurse-led protocol for ORIF ankle care where patients attended outpatient appointments at three defined stages: 10-14 days, 6 weeks and 12 weeks post-surgery. This protocol includes standardised procedures for each appointment, and the care is handed over to nurse-led clinics. It was expected by clinicians and staff at the fracture clinic that this method of care should both reduce the total number of return appointments and the cost of ORIF ankle care. The aim of this study was to investigate these hypotheses and provide a concrete cost comparison between the traditional method of care and the nurse-led protocol, having established that similar 
patient reported outcomes - health outcomes and patient satisfaction - can be achieved across both methods [4,5]. In their recent clinical guidelines on methods, evidence and recommendations for noncomplex fracture assessment and management (2015), the NICE identified no relevant economic evidence towards evaluating the most cost-effective mobilisation strategy in post-operative patients following ORIF of the ankle [4]. The use of nurse-led clinics in the UK has been growing since their endorsement by the UK government in the 1990s [6]. The evidence for their effectiveness is growing, and published literature has increasingly found them to improve health and patient outcomes as well as cost-effectiveness [7]. Although little research has previously been found evaluating cost-effectiveness of nurse-led clinics in secondary care, this evidence base is growing $[6,8]$.

\section{METHODS AND MODEL DEVELOPMENT}

The general research approach to this study was mixed methods research [9], which captures both qualitative and quantitative aspects of a system. This approach was implemented for this study due to the complexity of the system of interest.

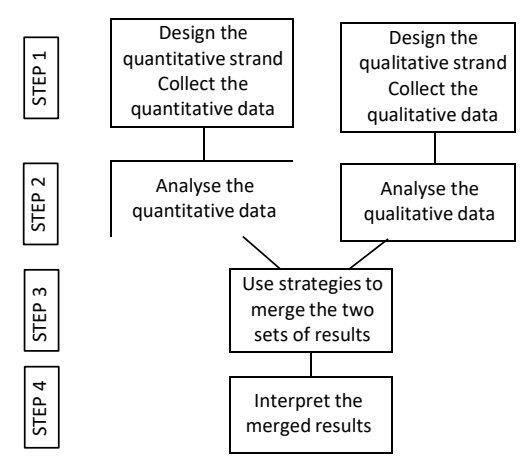

Figure 1: Steps involved in Mixed Methods research [9].

Concurrently, both qualitative and quantitative models were developed from two representative samples of data in order to produce a Discrete Event Simulation (DES) model for cost-modelling. The two representative samples of 50 ORIF ankle patients were selected from two Scottish fracture clinics Glasgow's Victoria Infirmary (VIC) fracture clinic, representing the traditional method of care, and the GRI fracture clinic, representing the protocol method of care, using systematic random sampling. These samples were found to be comparable in terms of patient demographics.

\subsection{Methods for evaluation}

In order to compare the total number of appointments attended per patient across the two systems, the non-parametric Mann-Whitney $U$ test for skewed data was used for statistical analysis. A systematic search of literature provided guidance towards adopting DES as the most appropriate costmodelling tool for this evaluation. The main advantage of simulation methods is that they are based on variability, hence they are more suited to modelling processes involving patient-flow [10]. DES has been used to develop detailed simulation models allowing application of a stochastic approach within the healthcare setting.

In this study DES was used to model and analyse costs with an activity-based costing approach, particularly for resources utilised. By using statistical distributions for activity durations and routings through complex pathways, activity costs are modelled more accurately. Specifically, SIMUL8 DES software was used for this study. Further to this, the literature was reviewed in order to appropriately select costs which were to be included in the evaluation, based on the viewpoint for the analysis and number of programmes being compared, among other criteria [11].

\section{RESULTS}

Through statistical analysis it was found that the difference in total number of appointments between the two groups was not statistically significant ( $p$ value $>0.05$ ). However, results generated through the DES models indicate that a total average saving of $£ 7,571.84$ for the care of a one-year period of ORIF ankle surgery patients (159 surgeries) can be achieved by implementing the nurse-led protocol. Overall this is a $28.12 \%$ reduction in costs from its implementation compared to the traditional method. The costs included in the evaluation can be seen in Table 1 along with the full results.

Table 1: Cost-modelling results from DES model after sufficient runs to achieve a 95\% confidence interval of results - total average costs for the care of a one-year period of ORIF ankle surgery patients

\begin{tabular}{|l|c|c|c|c|}
\hline \multirow{2}{*}{ Cost } & $\begin{array}{l}\text { Traditional } \\
\text { Model }\end{array}$ & $\begin{array}{l}\text { Protocol } \\
\text { Model }\end{array}$ & \multicolumn{2}{c|}{$\begin{array}{c}\text { Difference in cost between } \\
\text { traditional and protocol }\end{array}$} \\
\cline { 2 - 5 } & Average & Average & Difference & $\%$ Difference \\
\hline$X$-ray total cost & $£ 16,618.35$ & $£ 11,457.92$ & $-£ 5,160.44$ & $-31.05 \%$ \\
\hline Nurse staffing cost & $£ 2,718.48$ & $£ 5,997.06$ & $£ 3,278.57$ & $120.60 \%$ \\
\hline Orthopaedic consultant staffing cost & $£ 7,082.61$ & $£ 1,334.35$ & $-£ 5,748.26$ & $-81.16 \%$ \\
\hline Typist staffing cost & $£ 260.49$ & $£ 260.74$ & $£ 0.25$ & $0.10 \%$ \\
\hline Receptionist staffing cost & $£ 249.51$ & $£ 307.54$ & $£ 58.03$ & $23.26 \%$ \\
\hline Total cost & $\mathfrak{£ 2 6 , 9 2 9 . 4 4}$ & $£ 19,357.60$ & $\mathbf{- £ 7 , 5 7 1 . 8 4}$ & $\mathbf{- 2 8 . 1 2 \%}$ \\
\hline
\end{tabular}


It can be observed that nursing costs are increased with the implementation of the nurse-led protocol while orthopaedic consultant's costs are reduced, as expected. Some of the costs included in the evaluation did not change significantly across the two alternatives, however, they have been included for completeness. Total costs per patient could not be produced due to limitations further discussed.

\section{DISCUSSION AND LIMITATIONS}

The cost evaluation carried out in this study suggests that implementing a nurse-led protocol for ORIF ankle care is associated with significant savings in total staffing and X-ray costs compared to the traditional method of care. Hence, implementing this protocol may be an attractive option for other fracture clinics currently following the traditional method, supported by the facts that there is no difference in patient reported outcomes [4,5] and little to no implementation costs apart from minimal training, as nurses are already familiar with processes involved. However, it must be noted that convincing evidence is not sufficient for implementation, as medical practises differ in their organisation and workforce, among other differences. Hence care should be taken in evaluating the applicability of an intervention practise by practise.

Although these are encouraging results, further work is required in order to investigate the aftermath of the intervention with regards to what ways consultants' time is or can be used now that it has been freed up as well as the effects of increased workload on nursing staff.

Additionally, there are some limitations to this research. Firstly, the DES model used for costevaluation assumes that the nurse-led protocol is always followed with regards to $\mathrm{X}$-rays. This may not always be the case, hence further work would be required to obtain data on this and refine the costevaluation. Due to time and software restrictions, the cost-evaluation was limited to reporting total average costs. Ideally, an average cost per patient should be reported, however, the distribution of costs was unknown, hence it would not be appropriate to report an average cost per patient, as statistically it may not be an accurate measure of centre. Hence, future work would be required to evaluate a cost per patient.

\section{ACKNOWLEDGEMENTS}

This research was facilitated by Gillian Anderson, $\mathrm{Dr}$ Lech Rymaszewski and Margaret Nugent (GRI fracture clinic), as well as other GRI fracture clinic staff. Their insight and assistance were greatly appreciated in carrying out this research.

\section{REFERENCES}

[1] Jenkins, P., Morton, A., Anderson, G., Van Der Meer, R. and Rymaszewski, L. (2016). Fracture clinic redesign reduces the cost of outpatient orthopaedic trauma care. Bone and Joint Research, 5(2), pp.33-36.

[2] Hoffmann, C., Stoykova, B., Nixon, J., Glanville, J., Misso, K. and Drummond, M. (2002). Do Health-Care Decision Makers Find Economic Evaluations Useful? The Findings of Focus Group Research in UK Health Authorities. Value in Health, 5(2), pp.71-78.

[3] Spurgeon, P., Cooper, C. and Burke, R. (2012). The Innovation Imperative in Health Care Organisations: Critical Role of Human Resource Management in the Cost, Quality and Productivity Equation. Cheltenham: Edward Elgar Publishing, p.18.

[4] National Clinical Guideline Centre, (2015). Diagnosis, management and follow-up of noncomplex fractures: Clinical guidelines on methods, evidence and recommendations August 2015. National Institute for Health and Care Excellence, pp.234-240.

[5] McGlynn, J.M., Young, P.S., Miller, R.J., Kumar, C.S. (2012). Nurse-led review clinic for patients with ankle fractures treated operatively: audit of quality of service and patient satisfaction. GLAMOR meeting, Glasgow, April 2012.

[6] Williams, S., Williams, J., Tcherveniakov, P. and Milton, R. (2012). Impact of a thoracic nurse-led chest drain clinic on patient satisfaction. Interact Cardiovasc Thorac Surg 14(6), pp. 729-733.

[7] Hudorovic, N. and Vicic-Hudorovic, V. (2012). eComment. Nurse-led clinics and costeffectiveness. Interact Cardiovasc Thorac Surg 14(6), pp. 733-734.

[8] Rafferty, S. (2002). Do nurses do it better? Thorax 57(8), pp. 659-660.

[9] Creswell, J. and Plano Clark, V. (2011). Designing and conducting mixed methods research. 2nd ed. Los Angeles: SAGE Publications.

[10] Benneyan, J. (1997). An introduction to using computer simulation in healthcare: patient wait case study. J Soc Health Syst. 5: 1-15.

[11] Drummond, M., O'Brien, B., Stoddart, G. and Torrance, G. (1997). Methods for the economic evaluation of health care programmes. 2nd ed. New York: Oxford University Press. 